\title{
Duration discrimination of empty and filled intervals marked by auditory and visual signals
}

\author{
SIMON GRONDIN \\ Université Laurentienne, Sudbury, Ontario, Canada
}

\begin{abstract}
Experiments 1 and 2 compared, with a single-stimulus procedure, the discrimination of filled and empty intervals in both auditory and visual modalities. In Experiment 1, in which intervals were about $250 \mathrm{msec}$, the discrimination was superior with empty intervals in both modalities. In Experiment 2, with intervals lasting about $50 \mathrm{msec}$, empty intervals showed superior performance with visual signals only. In Experiment 3, for the auditory modality at 250 msec, the discrimination was easier with empty intervals than with filled intervals with both the forced-choice (FC) and the single stimulus (SS) modes of presentation, and the discrimination was easier with the FC than with the SS method. Experiment 4, however, showed that at 50 and 250 msec, with a FC-adaptive procedure, there were no differences between filled and empty intervals in the auditory mode; the differences observed with the visual mode in Experiments 1 and 2 remained significant. Finally, Experiment 5 compared differential thresholds for four marker-type conditions, filled and empty intervals in the auditory and visual modes, for durations ranging from .125 to $4 \mathrm{sec}$. The results showed (1) that the differential threshold differences among marker types are important for short durations but decrease with longer durations, and (2) that a generalized Weber's law generally holds for these conditions. The results as a whole are discussed in terms of timing mechanisms.
\end{abstract}

The aim of the present study was to compare two conditions in duration discrimination: the discrimination of filled intervals and the discrimination of empty intervals. An interval is said to be filled when there is one given continuous signal between onset and offset; the signal's onset and offset mark the interval's beginning and end. An empty interval is a silent duration, that is, without stimulation, included within two sensory signals that mark the beginning and end of the interval. This filled-empty comparison has been neglected in the literature on the psychophysics of time.

A recent paper by Rammsayer and Lima (1991) provides a direct comparison of filled and empty auditory intervals. Their investigation was restricted to very short time intervals (about $50 \mathrm{msec}$ ). The authors reported that the differential thresholds were smaller (performance was better) with filled intervals than with empty intervals. This is consistent with a comparison of Abel's (1972a, 1972b) two studies on duration discrimination, one with filled intervals and the other with empty intervals.

This research was supported by a grant from the Natural Sciences and Engineering Research Council of Canada. I wish to express my gratitude to Stan Koren for his excellent technical assistance, to Lynn Metthé for her help in the data collection, and to David Leadbeater for his help with the English language. I also extend special thanks to Peter Killeen, Lester Krueger, and John Flowers for their help or comments in the preparation of the manuscript. Portions of the material included in this article were presented at the 33rd Annual Meeting of The Psychonomic Society in St. Louis in 1992. Correspondence concerning this paper should be addressed to Simon Grondin, Department of Psychology, Laurentian University, Ramsey Lake Road, Sudbury, ON, Canada P3E 2C6 (e-mail: sgrondin@nickel.laurentian.ca).
On the other hand, comparisons of other studies suggest that empty intervals may be easier to discriminate than filled intervals. For filled auditory intervals, Small and Campbell (1962) reported Weber fractions of about $15 \%$ to $20 \%$ for durations between 40 and $400 \mathrm{msec}$; Stott (in Fraisse, 1978) had Weber fractions between 10\% and $13 \%$ for durations between 400 and $2,000 \mathrm{msec}$; and Henry (1948) observed Weber fractions of about $20 \%$ for durations between 50 and $500 \mathrm{msec}$. For empty auditory intervals, Goodfellow (1934) reported a Weber fraction of $7 \%$ for a $1-\mathrm{sec}$ interval and Getty (1975) reported Weber fractions of about $5 \%$ to $7 \%$ for durations between 200 and $2,000 \mathrm{msec}$.

The goal of the present research was to provide new direct evidence on comparisons of performance with filled and empty intervals. The data presented here cover a wide range of durations and were collected with various methods; furthermore, the investigation is not restricted to auditory signals but is extended to visual signals.

\section{EXPERIMENT 1}

In this experiment, filled and empty intervals were compared for the discrimination of intervals with a midpoint of $250 \mathrm{msec}$. The comparison was made for two types of intervals, visual and auditory. The discrimination was done between intervals whose lengths were expected to produce responses that would be close to $75 \%$ correct, that is, close to the differential threshold.

\section{Method}

Subjects. Six 22- to 28-year-old volunteers participated in this experiment. They were paid $\$ 5$ per session. 
Apparatus and Stimuli. Each observer was seated in a chair in a dimly lit room and asked to respond either "short" or "long", by pressing the appropriate button. Beside each button, there was a small light providing feedback after each trial. The auditory signal, presented binaurally, was a $1-\mathrm{kHz}$ tone with an intensity recorded at $70 \mathrm{~dB}$ SPL. The visual signal consisted of a circular light-emitting diode situated at about $1 \mathrm{~m}$ in front of the subject. For empty intervals, both markers were 20 -msec stimulations. The experiment was controlled by an IBM microcomputer.

Procedure. The observer had to discriminate between a short and a long interval presented according to the single-stimulus method. For the auditory intervals, the short and the long lasted 241 and $259 \mathrm{msec}$, respectively; for the visual intervals, the short and the long were set at 225 and $275 \mathrm{msec}$. Each trial began with a 140 -msec auditory signal and, after a $1-\mathrm{sec}$ preparation period, the short or the long interval was presented. According to the blocks of trials, this interval was a continuous signal or an empty duration included between two markers, with the duration of these intervals as indicated above. The observer was given $4 \mathrm{sec}$ to respond and, whatever was the response time, $200 \mathrm{msec}$ after the response a 1.7sec visual feedback was delivered. After the feedback, there was a 1 -sec intertrial period.

In this experiment, there were five sessions, the first one being for training. Each session contained eight blocks of 50 trials with 25 short and 25 long intervals presented randomly. These eight blocks consisted of two identical cycles of four blocks. Each of these four blocks corresponded to one of four types of marker conditions: auditory-empty, auditory-filled, visual-empty, and visual-filled. During the last four sessions, the order of these four conditions was varied according to a Latin square. Between the blocks there was a $15-\mathrm{sec}$ pause. Each session lasted about 35 to $40 \mathrm{~min}$. The analysis was done on the basis of 400 judgments by each subject in each marker condition.

\section{Results and Discussion}

The individual and mean results in each condition are reported in Table 1 . In the auditory condition, the mean $(81.08 \%)$ with empty intervals is significantly higher than the mean $(69.63 \%)$ with filled intervals $[t(5)=2.87, p<$ $.05]$. In the visual mode, the mean $(82.17 \%)$ with the empty intervals is also significantly higher than the mean (76.88\%) observed with the filled intervals $[t(5)=5.16$, $p<.01]$.

The results of this first experiment are surprising considering those reported by Abel (1972a, 1972b) and those reported by Rammsayer and Lima (1991). Their results and those obtained here appear opposed. To explain this difference, it is necessary to look more closely at the methodologies. One difference is that both Abel (1972a,

Table 1

Individual and Mean Probability of Responding Correctly Under Each Condition of Experiment 1

\begin{tabular}{|c|c|c|c|c|}
\hline \multirow[b]{2}{*}{ Subject } & \multicolumn{2}{|c|}{ Auditory } & \multicolumn{2}{|c|}{ Visual } \\
\hline & Filled & Empty & Filled & Empty \\
\hline 1 & 74.50 & 95.25 & 89.25 & 92.75 \\
\hline 2 & 69.00 & 75.25 & 77.50 & 88.00 \\
\hline 3 & 69.50 & 90.50 & 80.25 & 89.00 \\
\hline 4 & 63.25 & 66.75 & 69.25 & 73.75 \\
\hline 5 & 67.75 & 66.50 & 69.25 & 77.25 \\
\hline 6 & 73.75 & 92.25 & 77.75 & 80.25 \\
\hline Mean & 69.63 & 81.08 & 76.88 & 82.17 \\
\hline
\end{tabular}

1972b) and Rammsayer and Lima (1991), respectively, used a set of comparison intervals for evaluating the individual $\Delta t_{75}$ and $70.7 \%$ difference thresholds. In the present experiment, the discrimination was done between two fixed values. Another difference is that both Abel (1972a, 1972b) and Rammsayer and Lima (1991) used a forced-choice mode of presentation, whereas the method we employed here was the single stimulus. Moreover, in the only experiments designed explicitly for testing the differences between discrimination with empty and filled intervals, Rammsayer and Lima used different signals for empty and filled durations. Their empty intervals were marked by 3 -msec clicks, whereas their filled intervals were $1-\mathrm{kHz}$ tones. Finally, the investigation on this question by Rammsayer and Lima was limited to a $50-\mathrm{msec}$ standard duration. The following experiments were designed to test some of these methodological elements, which could be significant factors influencing discrimination.

\section{EXPERIMENT 2}

In this second experiment, the goal was to verify if the difference between empty and filled intervals found by Rammsayer and Lima (1991) with 50-msec intervals could also be observed with the single-stimulus mode of presentation. This verification was tested with both auditory and visual intervals.

\section{Method}

Subjects. Six 18- to 32-year-old volunteers participated in this experiment. They were paid $\$ 5$ per session.

Procedure. The apparatus and stimuli were the same as in Experiment 1 and the procedure was identical to that of Experiment 1. One thing was changed: the values of the short and long intervals. In the auditory mode, the parameters were set on the basis of the results reported by Rammsayer and Lima (1991), so that a general level of discrimination of approximately $75 \%$ of correct responses was expected, at least with the filled condition. Thus, the short and long intervals were set at 50 and $62 \mathrm{msec}$, respectively; in the visual modality, they were set at 50 and $80 \mathrm{msec}$. The short interval was set at $50 \mathrm{msec}$ because the standard was $50 \mathrm{msec}$ in Rammsayer and Lima and their comparators were greater than $50 \mathrm{msec}$.

\section{Results and Discussion}

The individual and mean results in each condition are reported in Table 2 . In the auditory modality, the mean $(78.28 \%)$ with the filled intervals is not significantly higher than the mean $(76.21 \%)$ with the empty intervals $[t(5)=1.05, p=.341]$. In the visual modality, the mean $(83.91 \%)$ with the empty intervals is significantly higher than the mean $(75.58 \%)$ with the filled intervals $[t(5)=$ $5.76, p<.011$.

The results of this experiment are somewhat surprising since they differ from those of the previous experiment. What is most surprising is the fact that what was observed at $250 \mathrm{msec}$, that is, a superiority of discrimination with empty intervals in both modalities, is observed here only in the visual modality. These results suggest that there might be differences in the nature of the temporal process involved at 50 and $250 \mathrm{msec}$. Indeed, 
Table 2

Individual and Mean Probability of Responding Correctly Under Each Condition of Experiment 2

\begin{tabular}{cccccc}
\hline & \multicolumn{2}{c}{ Auditory } & & \multicolumn{2}{c}{ Visual } \\
\cline { 2 - 3 } Subject & Filled & Empty & & Filled & Empty \\
\hline 1 & 83.17 & 84.00 & & 81.75 & 91.75 \\
2 & 70.75 & 71.00 & & 67.50 & 81.50 \\
3 & 72.75 & 74.75 & & 75.25 & 82.75 \\
4 & 83.75 & 72.50 & & 68.75 & 76.00 \\
5 & 77.75 & 74.75 & & 76.00 & 84.00 \\
6 & 81.50 & 80.25 & & 84.25 & 87.50 \\
Mean & 78.28 & 76.21 & & 75.58 & 83.91 \\
\hline
\end{tabular}

$50 \mathrm{msec}$ is a range of duration where some sensory factors are more likely to interfere with the processing of the duration (Allan \& Kristofferson, 1974).

On the other hand, the present results for the auditory modality at least are more in agreement with those of Rammsayer and Lima (1991) than with those of Experiment 1. In Experiment 2, the auditory discrimination tends to be better with filled intervals than with empty intervals. However, the difference observed here is very much weaker than the one reported by Rammsayer and Lima. This difference might depend on the utilization of different types of presentation of the stimuli, that is, forced-choice (FC) or single stimuli (SS). If this is the case, the benefit a subject could gain from the FC method should be apparent only with filled intervals, not with the empty ones. This hypothesis was tested in the next experiment.

\section{EXPERIMENT 3}

The goal of Experiment 3 was to test one factor that was likely to have produced the differences observed in Experiment 1 with empty versus filled intervals. This time, the comparison was made between empty and filled intervals with both methods of presentation, the FC and the SS methods. This investigation was here restricted to the auditory modality and to the $250-\mathrm{msec}$ intervals.

\section{Method}

Subjects. Fourteen 20- to 29-year-old volunteers participated in this experiment. They were paid $\$ 5$ per session.

Procedure. The apparatus and stimuli were as in the previous experiments. For the conditions where the SS method was employed, the trials were identical to those described earlier. For the FC method, the warning signal, preparation period, response period, feedback, and intertrial intervals were as for the SS method, but both stimuli, the short and the long, were presented within each trial with a 1 -sec interstimulus interval. The order of presentation of the short and the long, first or second, was random but equiprobable within each block. As in the previous experiments, there were eight blocks of 50 trials each.

In the present experiment, there were five sessions, one for familiarization and four experimental. In the familiarization session, there were two blocks of each of the four experimental conditions: FC-empty, FC-filled, SS-empty, and SS-filled. There was only one condition per experimental session. We adopted the procedure of having the experimental conditions in different sessions, which was not the case in Experiments 1 and 2, in order to avoid an immediate representation of the short and the long intervals, filled or empty, when passing from the SS method to the FC method or from the FC method to the SS method. The order of presentation of the conditions was varied according to a Latin square resulting in four cycles: 4 subjects were assigned to Cycle 1 and 4 were assigned to Cycle 2; 3 subjects were assigned to Cycle 3 and 3 were assigned to Cycle 4.

For all four experimental conditions, the short and long intervals were set, as in Experiment 1, at 241 and $259 \mathrm{msec}$.

\section{Results and Discussion}

The individual and mean results in each condition are reported in Table 3 . The difference between the means of each condition was tested with a randomized block factorial ANOVA ( 2 methods $\times 2$ types of intervals; Kirk, 1982). The results show a significant difference between the empty- and filled-interval conditions $[F(1,39)=6.18$, $p<.05]$ and between the FC and SS methods $[F(1,39)=$ $6.86, p<.05]$. The interaction effect was not significant $[F(1,39)=.26]$.

There are two aspects of interest in this experiment. First, the difference between the filled and the empty intervals reported in Experiment $\mathbf{l}$ for the auditory intervals was replicated here. The second aspect, more important in our effort to search for the sources of difference between the results of Rammsayer and Lima (1991) and those of the present study, concerns the possible effects of the FC and SS procedures. It was expected that using the FC method instead of the SS method would provide a greater benefit for the filled intervals than for the empty intervals. In brief, an interaction effect was expected, but was not observed. Thus, the difference between the FC and SS procedures cannot account for the difference between our results and those of Rammsayer and Lima.

Experiment 3 is notable because it shows better performance with the FC procedure than with the SS procedure. Usually, in psychophysical tasks using dimensions

Table 3

Individual and Mean Probability of Responding Correctly Under Each Condition of Experiment 3

\begin{tabular}{|c|c|c|c|c|}
\hline \multirow[b]{2}{*}{ Subject } & \multicolumn{2}{|c|}{ Forced Choice } & \multicolumn{2}{|c|}{ Single Stimulus } \\
\hline & Filled & Empty & Filled & Empty \\
\hline 1 & 67.75 & 66.00 & 62.72 & 64.67 \\
\hline 2 & 79.50 & 88.25 & 71.68 & 84.70 \\
\hline 3 & 74.75 & 66.25 & 65.92 & 70.93 \\
\hline 4 & 72.25 & 77.00 & 54.88 & 75.40 \\
\hline 5 & 66.00 & 84.25 & 58.25 & 84.50 \\
\hline 6 & 57.00 & 69.50 & 57.39 & 67.00 \\
\hline 7 & 67.75 & 64.75 & 58.69 & 59.50 \\
\hline 8 & 64.25 & 86.00 & 62.47 & 80.25 \\
\hline 9 & 62.55 & 62.75 & 62.00 & 67.92 \\
\hline 10 & 94.75 & 86.00 & 88.25 & 78.95 \\
\hline 11 & 74.00 & 76.75 & 83.00 & 66.58 \\
\hline 12 & 72.04 & 66.25 & 71.25 & 63.41 \\
\hline 13 & 83.50 & 86.25 & 67.67 & 66.41 \\
\hline 14 & 69.75 & 76.00 & 62.00 & 72.25 \\
\hline Mean & 71.85 & 75.43 & 66.16 & 71.61 \\
\hline
\end{tabular}


other than time, the FC method leads to better performance than the SS method (see Creelman \& Macmillan, 1979; Green \& Swets, 1966). In the case of duration discrimination, Allan, Kristofferson, and Rice (1974) have reported data showing no significant differences between the FC and SS methods. Their investigation was conducted with very short intervals $(<100 \mathrm{msec})$ and in the visual modality. Indeed, they used a "dark flash," in which a visual display was illuminated but the duration stimulus was not. The absence of a difference between the FC and SS methods as reported by Allan et al. (1974) might be due to their utilization of different groups of subjects in each task. Allan et al. noted that Carbotte (1972) also reported an absence of difference between the SS and FC methods used by the same subjects in the discrimination of empty auditory intervals (these results are briefly reported in Carbotte, 1973).

\section{EXPERIMENT 4}

Since the method of presentation of the stimuli might change the evaluation of performance in a given markertype condition, the experimental conditions of Experiments 1 and 2 needed to be reexamined. These conditions were filled-empty, auditory-visual, $50 \mathrm{msec}$, and $250 \mathrm{msec}$. In this fourth experiment, an adaptive procedure, which involved FC conditions, was used.

\section{Method}

Subjects. Sixteen 19- to 32-year-old volunteers participated in this experiment. They were paid $\$ 10$ for their participation.

Procedure. The material in the present experiment was the same as that used in the previous experiments. The main change in the method was the utilization of an FC-adaptive procedure. One trial consisted of a presentation of both a standard duration and a comparator, in random order. The subject had to signal, by pressing the appropriate button, if the first interval was shorter or longer than the second interval. The empty intervals were marked by two 20-msec signals. The first and second intervals were separated by a 1-sec interval. A 1.7-sec feedback was provided to the subject, but there was no warning signal. The next trial started $2 \mathrm{sec}$ after presentation of the feedback.

With the FC-adaptive procedure, the difficulty of the discrimination was adjusted after each trial. More precisely, after each correct response, the comparator was decreased (i.e., brought nearer to the standard) by a factor of $X$, and, after each wrong response, the comparator was increased by a factor of $3 X$. This technique, a weighted up-down method, is reported to provide an estimation of the $\Delta t_{\text { }}$ (Kaernbach, 1991; Rammsayer, 1992b).

Each estimation of a $\Delta t_{75}$ involved 50 trials distributed in three blocks of 10, 20, and 20 trials. For each block, the steps had one given value. For the estimation with a 50-msec standard, the comparator was set at $98 \mathrm{msec}$ for the first trial and the steps for Blocks 1,2 , and 3 were set at 8,4 , and $2 \mathrm{msec}$, respectively. With the 250 -msec standard, the comparator was set at $500 \mathrm{msec}$ and the steps for Blocks 1, 2, and 3 were set at 30,10 , and $3 \mathrm{msec}$, respectively. Note that the computer program was arranged so that it was not possible for the comparator to have a value smaller than or equal to the value of the standard. Thus, there were some successive trials for which the value of the comparator was the same.

The subjects participated in two sessions, one for each standard value. In each session, there were four conditions: auditory-empty, auditory-filled, visual-empty, and visual-filled. The order of presentation of these conditions and the order for the 50 - and $250-\mathrm{msec}$ sessions were balanced among subjects. At the beginning of the first session, there were some practice trials to make sure the subjects understood the task.

\section{Results and Discussion}

In Experiment 4, the dependent variable of interest was the differential threshold, which here was estimated by subtracting the value of the standard from the average of the comparators in the last 10 trials. The lower the threshold, the better the performance. The comparisons of interest in this experiment were, as in the previous experiments, those between filled and empty intervals. Table 4 shows the individual and average differential

Table 4

Individual and Mean Differential Thresholds Under Each Condition of Experiment 4

\begin{tabular}{|c|c|c|c|c|c|c|c|c|}
\hline \multirow[b]{2}{*}{ Subject } & \multicolumn{4}{|c|}{$50 \mathrm{msec}$} & \multicolumn{4}{|c|}{$250 \mathrm{msec}$} \\
\hline & $\mathrm{Ae}$ & Af & $\mathrm{Ve}$ & $\mathrm{Vf}$ & $\mathrm{Ae}$ & Af & $\mathrm{Ve}$ & $\mathrm{Vf}$ \\
\hline 1 & 6.80 & 24.60 & 43.00 & 39.00 & 38.10 & 21.90 & 80.90 & 92.90 \\
\hline 2 & 12.60 & 10.60 & 14.20 & 22.20 & 6.80 & 59.70 & 76.90 & 73.70 \\
\hline 3 & 15.00 & 6.20 & 16.60 & 15.00 & 83.70 & 35.30 & 97.70 & 106.50 \\
\hline 4 & 14.20 & 11.00 & 46.20 & 43.00 & 94.10 & 20.90 & 40.90 & 130.50 \\
\hline 5 & 4.20 & 15.00 & 15.80 & 21.40 & 8.10 & 32.90 & 53.50 & 73.90 \\
\hline 6 & 15.40 & 8.60 & 22.20 & 27.70 & 84.10 & 48.10 & 80.10 & 137.70 \\
\hline 7 & 3.40 & 3.40 & 15.00 & 11.80 & 9.70 & 24.50 & 30.10 & 26.50 \\
\hline 8 & 5.40 & 16.60 & 19.80 & 24.60 & 66.50 & 114.10 & 75.70 & 85.30 \\
\hline 9 & 6.00 & 13.40 & 11.00 & 23.80 & 6.60 & 6.40 & 48.90 & 59.70 \\
\hline 10 & 19.00 & 3.40 & 14.00 & 9.00 & 40.50 & 12.30 & 27.50 & 42.90 \\
\hline 11 & 25.40 & 14.60 & 20.60 & 33.80 & 40.90 & 21.30 & 44.50 & 148.90 \\
\hline 12 & 8.60 & 6.20 & 14.20 & 19.80 & 20.10 & 21.70 & 18.50 & 70.50 \\
\hline 13 & 7.80 & 11.00 & 23.80 & 28.60 & 13.70 & 7.70 & 67.70 & 88.90 \\
\hline 14 & 12.60 & 16.60 & 12.40 & 29.40 & 33.70 & 49.30 & 104.50 & 96.50 \\
\hline 15 & 5.40 & 11.00 & 27.80 & 39.00 & 21.30 & 43.70 & 29.30 & 117.70 \\
\hline 16 & 7.80 & 8.60 & 20.80 & 24.60 & 12.60 & 9.00 & 28.60 & 43.00 \\
\hline Mean & 10.60 & 11.30 & 21.08 & 25.79 & 35.66 & 33.05 & 56.58 & 87.19 \\
\hline
\end{tabular}

Note-Ae $=$ auditory-empty, Af $=$ auditory-filled, $\mathrm{Ve}=$ visual-empty, and $\mathrm{Vf}=$ visual-filled. 
thresholds for each experimental condition. Note that the individual differences are very prominent. This might be due to the fact that there was only one estimation for each subject, for each experimental condition. At $50 \mathrm{msec}$, the differential threshold for the filled intervals was higher than the differential threshold for the empty intervals in the visual mode $[t(15)=2.80, p<.05]$ but not in the auditory mode $[t(15)=.32$, n.s. $]$. At $250 \mathrm{msec}$, once again performance was significantly better with the empty intervals than with the filled intervals in the visual mode $[t(15)=3.39, p<.01]$ but not with the auditory mode $[t(15)=.32$, n.s. $]$.

Following this fourth experiment, it was now clearer that sensory mode is a key factor affecting performance differences with filled and empty intervals. With the visual mode, the superior discrimination with the empty intervals reported here is consistent with the results reported in Experiments 1 and 2. With the auditory mode, the results at $50 \mathrm{msec}$ are consistent with those of Experiment 2 but not with those of Rammsayer and Lima (1991). At $250 \mathrm{msec}$, the present auditory results are inconsistent with those of Experiments 1 and 3.

Let us first consider the case of the 50-msec intervals. Although there are some slight methodological differences between the procedure of Experiment 4 and the one used by Rammsayer and Lima (1991), a direct comparison of the results is reasonable. One might observe that the main difference between our differential thresholds and theirs is not for filled intervals (about $11 \mathrm{vs} .7 \mathrm{msec}$ ) but for empty intervals (about $11 \mathrm{vs.} 21 \mathrm{msec}$ ). Indeed, the results of Experiment 4 with empty intervals are far better than Rammsayer and Lima's. This might well be due to the fact that Rammsayer and Lima used 3-msec clicks for marking the empty intervals, whereas the markers in the present experiment were 20 -msec tones. In this context, Klumpp and Eady (1956), as noted by Rammsayer and Lima, have reported the interaural time difference thresholds to be $11 \mu \mathrm{sec}$ with $1-\mathrm{kHz}$ tones but $28 \mu \mathrm{sec}$ with clicks. An effect based on the quality of the auditory signal might well apply to the discrimination of very short time intervals. Indeed, for the discrimination of very short empty time intervals, the variations of frequency, and also of intensity, are known to produce important effects on performance (Divenyi \& Danner, 1977; Divenyi \& Sachs, 1978).

At $250 \mathrm{msec}$ in the auditory mode, the results of both Experiment 1 and Experiment 3 were better with the empty intervals; this was not the case in Experiment 4. On the basis of the results of Experiment 3, the disappearance of the superior performance with empty intervals cannot be attributed to the use of the FC procedure. Indeed, what is particular to the adaptive procedure is not only the utilization of the FC procedure, but also the fact that the comparator is changed from trial to trial, which was not the case in Experiment 3. In Experiment 3, the use of only two intervals, one short and one long, could have contributed to forming a steady and reliable representation of the short and long intervals. This represen- tation could be easier to maintain in the case of empty intervals than in the case of filled intervals, because the empty intervals, when the signals are provided within one sensory mode, might take advantage of some grouping, or perceptual organization (Fraisse, 1952). This speculative explanation would be more effective with the auditory mode than with the visual mode, if we assume that in the latter case other sources of variance are more important in explaining discrimination performance. This assumption is reasonable since the differential thresholds are much higher, both at $50 \mathrm{msec}$ and at $250 \mathrm{msec}$, in the visual mode than in the auditory mode. Better performance with auditory markers than with visual markers has also been reported elsewhere (Grondin \& Rousseau, 1991; Rousseau, Poirier, \& Lemyre, 1983).

\section{EXPERIMENT 5}

Experiment 5 was designed to clarify one key question in the time-perception field: how does the differential threshold, here defined as $\Delta t_{75}$, vary with duration? Not only did this experiment cover a wide range of durations, but it also provided an opportunity for comparing these variations in conditions in which the marking of intervals differed: filled and empty intervals, with auditory and visual stimulations. Such an investigation of the variation of differential thresholds with duration, with the same subjects and with different marker-type conditions, has remained a neglected question in the literature on the psychophysics of time. The investigation here was made using two methods. For 3 subjects, the differential thresholds were estimated with a method that was a variation of the SS method, the many-to-few method (Allan, 1979); for 1 subject, the differential thresholds were estimated with an adaptive procedure.

\section{Method}

Subjects. Three 23- to 30-year-old volunteers participated in the 24 sessions of this experiment with the many-to-few method. They were paid $\$ 5$ per session. Subject 3 was a highly skilled pianist. Subject 4 , the author, was tested with the adaptive procedure.

Procedure A: Psychometric functions. The apparatus and stimuli were as in Experiment 4. With the many-to-few method, the course of a trial was identical to that in Experiments 1 and 2. There were six identical blocks of 60 trials per session. When the many-to-few method was used in the experiment, there were more than one short and one long duration presented to the subject. Indeed, six durations were presented 10 times in a random order within each block. Three of these durations were shorter $(-1,-2,-3)$ and three were longer $(+1,+2,+3)$ than a midpoint value. The short and long intervals were doubled each time the midpoint value was doubled. This choice of parameters applied for each marker-type interval. The parameters for each midpoint value are reported in Table 5 . The midpoint values were $.125, .25, .5,1,2$, and $4 \mathrm{sec}$.

For each subject, 24 differential thresholds were estimated: 4 marker-type conditions $\times 6$ midpoint values. These 4 marker types were "empty auditory-auditory" (Ae), "filled auditory"' (Af), "empty visual-visual" (Ve), and "filled visual" (Vf). Each differential threshold, here defined as the difference between a short and a long interval necessary for reaching $75 \%$ of correct responses $\left(\Delta t_{73}\right)$, was based on 360 judgments. Subject 1 did the 24 sessions 
Table 5

Choice of Short and Long Intervals in Each Marker-Type Condition and for Each Midpoint Value With the Many-to-Few Procedure of Experiment 5

\begin{tabular}{|c|c|c|c|c|c|c|}
\hline \multirow{2}{*}{$\begin{array}{c}\text { Comparison } \\
\text { Stimulus } \\
\text { Values }\end{array}$} & \multicolumn{6}{|c|}{ Midpoint Value (msec) } \\
\hline & 125 & 250 & 500 & 1,000 & 2,000 & 4,000 \\
\hline \multicolumn{7}{|c|}{ Auditory (Filled and Empty) } \\
\hline-3 & 115 & 230 & 460 & 920 & 1,840 & 3,680 \\
\hline-2 & 119 & 238 & 476 & 952 & 1,904 & 3,808 \\
\hline-1 & 123 & 246 & 492 & 984 & 1,968 & 3,936 \\
\hline+1 & 127 & 254 & 508 & 1,016 & 2,032 & 4,064 \\
\hline+2 & 131 & 262 & 524 & 1,048 & 2,096 & 4,192 \\
\hline+3 & 135 & 270 & 540 & 1,080 & 2,160 & 4,320 \\
\hline \multicolumn{7}{|c|}{ Visual (Filled and Empty) } \\
\hline-3 & 105 & 210 & 420 & 840 & 1,648 & 3,360 \\
\hline-2 & 113 & 226 & 452 & 904 & 1,808 & 3,616 \\
\hline-1 & 121 & 242 & 484 & 968 & 1,936 & 3,872 \\
\hline+1 & 129 & 258 & 516 & 1,032 & 2,064 & 4,128 \\
\hline+2 & 137 & 274 & 548 & 1,096 & 2,192 & 4,384 \\
\hline+3 & 145 & 290 & 580 & 1,160 & 2,320 & 4,640 \\
\hline
\end{tabular}

in the following order: Ae, Af, Ve, and Vf at $.125 \mathrm{sec}$, then at $.25, .5,1,2$, and $4 \mathrm{sec}$. The other 2 subjects did the sessions in the reverse order, beginning at $4 \mathrm{sec}$ and ending at $.125 \mathrm{sec}$, and, for each midpoint value, beginning with $\mathrm{Vf}$ and finishing with $\mathrm{Ae}$. Subject 3 did two of the conditions (Ae-.25 and Ve-.5) a second time because her first session showed very low differential thresholds. For Subject 3, the second evaluation of the thresholds is the value reported here.

Before each session, the subject was first presented 10 examples of the midpoint value. For example, for a session where the midpoint was $.125 \mathrm{sec}$, the subject was presented a .125-sec interval 10 times. The shortest sessions $(.125 \mathrm{sec})$ lasted about $30 \mathrm{~min}$ and the longest $(4 \mathrm{sec})$ about $60 \mathrm{~min}$.

Procedure B: Adaptive. Essentially, this method was the same as that in Experiment 4: the interstimulus intervals, the number of blocks and trials per blocks, and the adjustments by 3 times the value of the steps for wrong responses were identical. In the present case, there were six standards of interest: $.125, .25, .5,1,2$, and $4 \mathrm{sec}$. For each standard, two types of sessions were adopted for estimating the differential thresholds, one with ascending values for the comparator and one with descending values. For each type, the threshold was evaluated twice. The mean of the best score for each type was kept as the estimation of the differential threshold. For each standard, the values of the comparators and of the steps in each block, for both descending and ascending method, are reported in Table 6.

The subject was conducted through the different conditions (96 estimations: 2 ascending series and 2 descending series $\times 4$ markertype conditions $\times 6$ standards) in the following order: $24(4 \times 6)$ estimations were completed with the descending series, followed by 24 with the ascending series, 24 with the descending series, and 24 in the ascending series. For each of these series of 24 , the order of the standard was random and, preceding the next standard, an estimation was made for each of the four marker types. The order for these four conditions was random.

\section{Results and Discussion}

For each of the 24 conditions with the many-to-few procedure, a 6-point psychometric function was traced, plotting the six comparison durations (from short $[-3]$ to long $[+3]$ ) on the $x$ axis and the $Z$ transformation of the probability of responding "long" on the $y$ axis. " The differ- ential threshold was then calculated as in the conventional constant-stimuli method. Two $x$ values corresponding to $75 \%(Z=.67)$ and $25 \%(Z=-.67)$ of long responses were estimated, and their difference, divided by 2 , was the estimated differential threshold. These two points, $75 \%$ and $25 \%$, represent the midpoint between a zero level of discrimination $(50 \%)$ and a perfect level of discrimination $(100 \%$ and $0 \%)$.

Table 7 reports the differential threshold value $\left(\Delta t_{75}\right)$, the point of subjective equality (PSE), and the Weber fraction $\left(\Delta t_{75} / T\right)$ of each subject in each of the 24 experimental conditions. The PSE is the value for which the internal duration appears equal, on average, to the midpoint for one given condition. For Subjects 1,2 , and 3 , all the $R^{2}$ exceeded $.88, .93$, and .90 , respectively.

For Subject 4, the differential threshold was calculated by taking the absolute value of the difference between the standard and the mean value of the comparators for the last 10 trials. This should provide an estimation of $\Delta t_{75}$ (Kaernbach, 1991; Rammsayer, 1992b). As noted earlier, the differential thresholds reported here are the mean of the best performance (lower threshold) in each ascending and descending series. The Weber fraction $\left(\Delta t_{75} / T\right)$ for each condition is reported in Figure 1d.

Figure 1 shows the discrimination function of each subject in each condition. In these, $\Delta t_{75} / T$ is plotted as a function of $T$. For most individual functions, the pattern is the same: the Weber fraction, as expressed by $\Delta t_{75} / T$, increases for lower values of $T$, the midpoint values. This is consistent with a generalized form of Weber's law, which states that the variability of discrimination judgments is proportional to stimulus magnitude plus a constant that is independent of the stimulus magnitude. In the present case, the stimulus is duration. Note that for Subject 3 in the Af condition, the Weber fractions remain constant.

Different models could be employed for describing the relation between the differential threshold and $T$. A first and simple description is actually provided by the generalized Weber's law $\left(\Delta t_{75}=k T+a\right)$. For each subject, and for each marker-type condition, the best fitting values for $k$ and $a$ were estimated for the generalized Weber's law. The values are reported in Table 8 . The slope is the estimation of $k$ and is here an estimation of the Weber fraction. Of note here is that, for 1 given subject, the Weber

Table 6

Parameters Used With the Adaptive Procedure of Experiment 5 (Subject 4)

\begin{tabular}{lrrrrrr}
\hline & \multicolumn{6}{c}{ Standard (msec) } \\
\cline { 2 - 7 } Step Trials & 125 & 250 & 500 & 1,000 & 2,000 & 4,000 \\
\hline $1-10$ & 12 & 25 & 50 & 100 & 200 & 400 \\
$11-30$ & 5 & 10 & 15 & 20 & 25 & 30 \\
$31-50$ & 2 & 3 & 4 & 5 & 6 & 7 \\
& & & & & & \\
First Comparator & & & & & & \\
Ascending Series & 190 & 375 & 750 & 1,500 & 3,000 & 6,000 \\
Descending Series & 60 & 125 & 250 & 500 & 1,000 & 2,000 \\
\hline
\end{tabular}




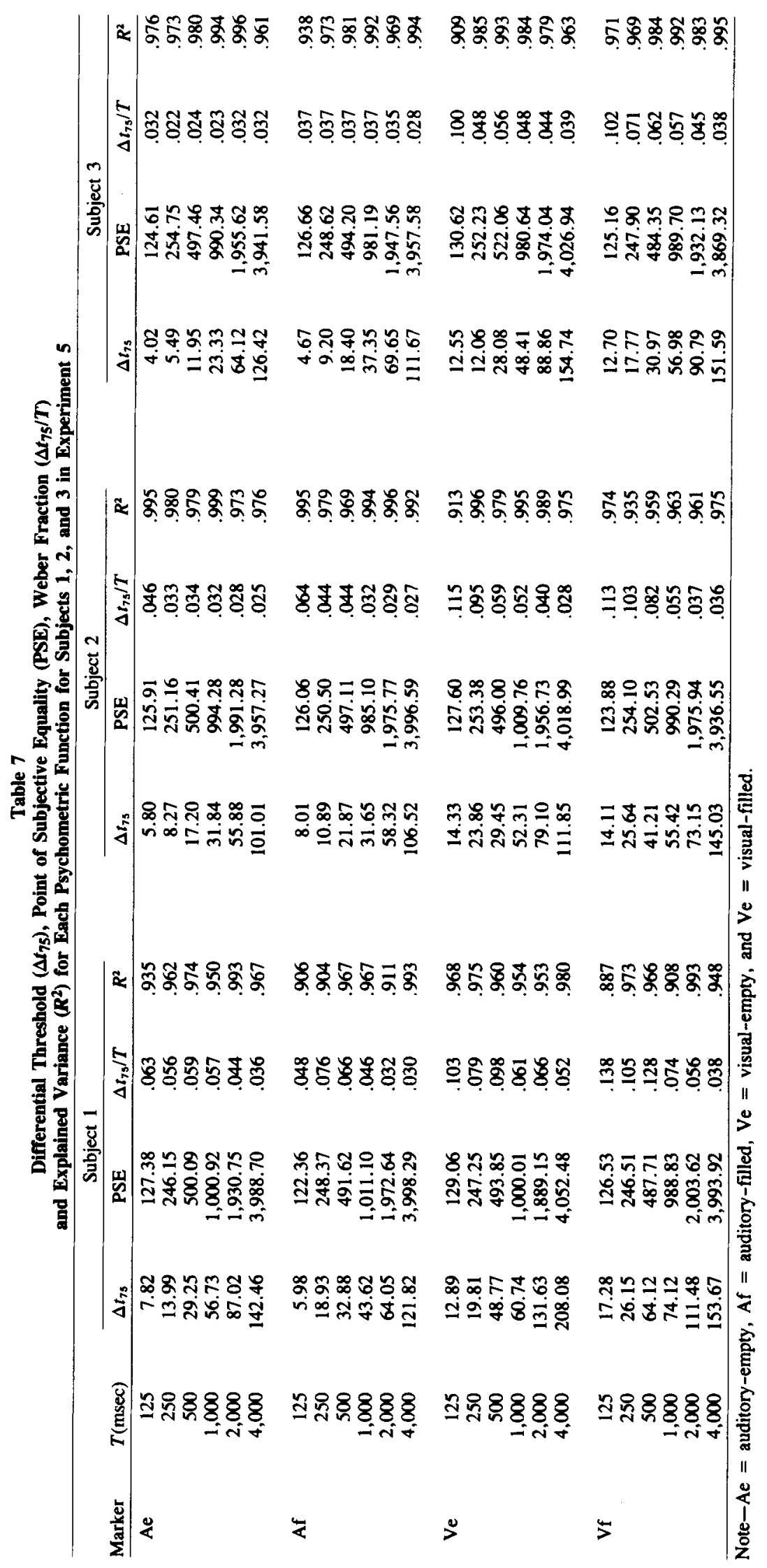




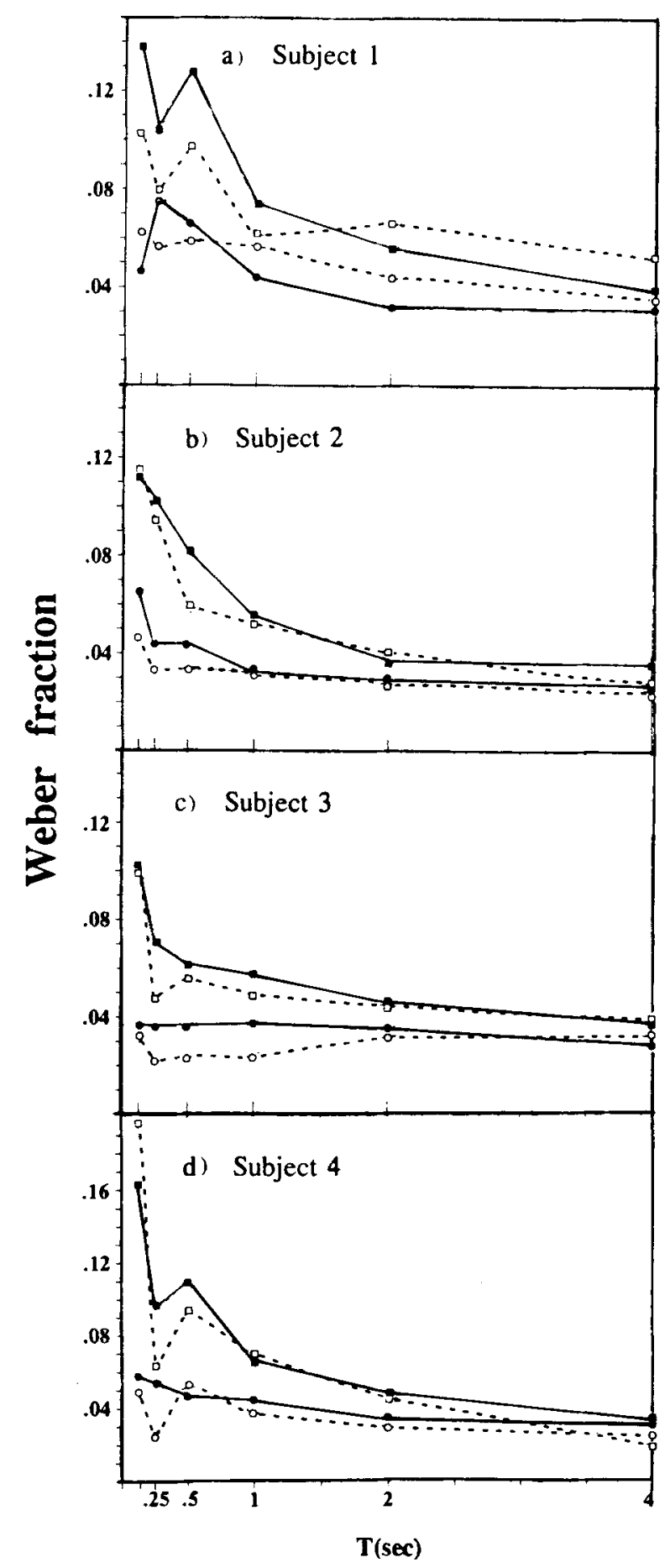

Figure 1. Individual Weber fractions $\left(\Delta t_{75} / T\right)$ for each subject of Experiment 5 plotted as a function of the base duration, $T$. Open circles $=$ empty auditory intervals, filled circles $=$ filled auditory intervals, open squares = empty visual intervals, filled squares $=$ filled visual intervals.

fraction remains quite similar for the different markertype conditions. The main differences among the markertype conditions are located in the value of the intercept, $a$. This parameter, often referred to in psychophysics as a background sensory noise, denotes that the difference between these marker-type conditions does not seem to depend on a timing mechanism (Weber fraction, $k$ ), but on another source of variance. The amount of variance here is mainly related to the sensory mode, not to the fact that the intervals are filled or empty.

Another description for the relation between the differential threshold, or the just noticeable difference (jnd), and $T$ could be provided by the following equation: jnd = $k T^{b}$. In such a power relation, the parameter $b$ is of interest. Krueger (1989) has reported that the deviation from Weber's law could be expressed in terms of $b$. A value of $b=1$ would be expected from Weber's law. The deviation from Weber's law found in the auditory area typically results in estimations of $b$ to be around .9 (Krueger, 1989). The estimation of $b$ for each marker-type condition for each subject is reported in Table 9. These values were calculated by an estimation of the slope of the bestfitting line between the log of jnd plotted as a function of the $\log$ of $T$. The explained variance is generally very good for each function. The values of $b$ are smaller (i.e., the deviations from Weber's law are greater) for the visual mode than for the auditory mode, and are smaller with the filled intervals than with the empty intervals in the auditory mode.

Another model for analyzing the variability of temporal judgments as a function of the duration to be timed is provided by Killeen and Weiss (1987). This model is a general one that may be used by any theories of energy detection relying on neural counting mechanisms. Indeed, the description of the model was first reported for the interpretation of the case of temporal discriminations.

In brief, Killeen and Weiss's model (1987) states that given the average duration of an entire interval $t$ (or $\mu_{\mathrm{T}}$ ), the average number of subintervals $n$ (or $\mu_{\mathrm{N}}$ ), and the average duration of a subinterval $d$ (or $\mu_{\mathrm{D}}$ ),

$$
t=n d
$$

and

$$
\sigma_{\mathrm{T}}^{2}=n \sigma_{\mathrm{D}}^{2}+d^{2} \sigma_{\mathrm{N}}^{2} .
$$

The total variance is then a combination of errors coming from the weighted sum of two sources, $\sigma_{\mathrm{D}}^{2}$ and $\sigma_{\mathrm{N}}^{2}$. The growth of these variances is assumed to correspond to these equations:

$$
\sigma_{\mathrm{D}}^{2}=\alpha_{2} d^{2}+\alpha_{1} d+\alpha_{0}
$$

and

$$
\sigma_{\mathrm{N}}^{2}=\beta_{2} n^{2}+\beta_{1} n+\beta_{0} .
$$

Killeen and Weiss referred to Equations 3 and 4 as the fundamental error equations. In cases where $\alpha_{0}>0$ and $\alpha_{2}>0$, the optimal duration for the subintervals $\left(d^{*}\right)$ is

$$
d^{*}=\left[\alpha_{0} /\left(\alpha_{2}+\beta_{1}\right)\right]^{1 / 2} \text {. }
$$

The accuracy of performance in a timing task could be predicted by inserting Equation 5 into Equation 2. This results in an equation where the variance in the estimates of a time interval is a quadratic function of this interval: 
Table 8

Individual Slope, Intercept, and Explained Variance $\left(\boldsymbol{R}^{\mathbf{2}}\right)$ When the Differential Thresholds Are Plotted as a Function of Time (Experiment 5)

\begin{tabular}{|c|c|c|c|c|c|c|c|c|c|c|c|c|}
\hline \multirow[b]{2}{*}{ Condition } & \multicolumn{3}{|c|}{ Subject I } & \multicolumn{3}{|c|}{ Subject 2} & \multicolumn{3}{|c|}{ Subject 3} & \multicolumn{3}{|c|}{ Subject 4} \\
\hline & Slope & Intercept & $R^{2}$ & Slope & Intercept & $R^{2}$ & Slope & Intercept & $R^{2}$ & Slope & Intercept & $R^{2}$ \\
\hline $\mathrm{Ae}$ & .034 & 11.130 & .977 & .025 & 4.394 & .996 & .032 & -3.313 & .996 & .023 & 8.660 & .965 \\
\hline Af & .028 & 11.598 & .981 & .025 & 6.379 & .998 & .028 & 5.279 & .983 & .029 & 8.344 & .991 \\
\hline $\mathrm{Ve}$ & .050 & 14.080 & .981 & .025 & 19.299 & .959 & .037 & 8.422 & .994 & .014 & 34.980 & .523 \\
\hline Vf & .033 & 30.761 & .913 & .031 & 17.868 & .981 & .036 & 13.222 & .988 & .028 & 29.326 & .920 \\
\hline
\end{tabular}

Note-Ae $=$ auditory-empty, Af $=$ auditory-filled, $\mathrm{Ve}=$ visual-empty, and $\mathrm{Vf}=$ visual-filled.

$$
\sigma_{\mathrm{T}}^{2}=A t^{2}+B t+C
$$

or

$$
\sigma_{\mathrm{T}}=\left(A t^{2}+B t+C\right)^{1 / 2} .
$$

The literature offers more details about these transformations (see Fetterman \& Killeen, 1990; Killeen, 1992; or Killeen \& Weiss, 1987).

We can use Equation 7 for analyzing the data of Experiment 5 . The analysis is made for each marker-type condition on the basis of the average data of the 4 subjects (Figure 2). Table 10 shows the best-fitting values for Equation 7. In these cases, parameter $C$ should be set at $0 . C$ reflects constant error in the counter (or $\beta_{0}$ ). The value for $\sqrt{ } A$ is the Weber fraction, or $\beta_{2}$, and Killeen and Weiss argue that this value is determined by counter error. The value of $B$ is made up of different factors ( $\alpha$ values in Equation 3, and $\beta_{1}$ in Equation 4).

Table 9

Estimation of the Exponent $b$, and Explained Variance $\left(\boldsymbol{R}^{2}\right)$, in the Power Functions Relating the

\begin{tabular}{|c|c|c|c|c|c|c|c|c|}
\hline \multirow[b]{3}{*}{ Subject } & \multicolumn{8}{|c|}{ Condition } \\
\hline & \multicolumn{2}{|c|}{$\mathrm{Ae}$} & \multicolumn{2}{|c|}{ Af } & \multicolumn{2}{|c|}{$\mathrm{Ve}$} & \multicolumn{2}{|c|}{ Vf } \\
\hline & $b$ & $R^{2}$ & $b$ & $R^{2}$ & $b$ & $R^{2}$ & $b$ & $R^{2}$ \\
\hline 1 & .85 & .992 & .78 & .947 & .82 & .985 & .66 & .958 \\
\hline 2 & .85 & .995 & .76 & .992 & .60 & .991 & .62 & .981 \\
\hline 3 & 1.04 & .992 & .93 & .996 & .79 & .983 & .74 & .995 \\
\hline 4 & .86 & .938 & .81 & .998 & .46 & .742 & .56 & .962 \\
\hline
\end{tabular}
Just Noticeable Difference and Time (Experiment 5)

Note-Ae $=$ auditory-empty, $\mathrm{Af}=$ auditory-filled, $\mathrm{Ve}=$ visual-empty, and $\mathrm{Vf}=$ visual-filled.

Table 10

Parameter Estimates and Explained Variance $\left(\boldsymbol{R}^{\mathbf{2}}\right)$ for Equation 7 for Mean Results of Experiment 5

\begin{tabular}{llcc}
\hline & \multicolumn{3}{c}{ Parameters } \\
\cline { 2 - 4 } \multicolumn{1}{c}{ Condition } & $\sqrt{ } A$ & $B$ & $R^{2}$ \\
\hline Auditory-empty & .041 & $1.056(1.626)$ & $.995(.992)$ \\
Auditory-filled & .038 & $1.674(1.732)$ & $.997(.997)$ \\
Visual-empty & .035 & $6.215(5.634)$ & $.984(.983)$ \\
Visual-filled & .033 & $7.414(6.559)$ & $.992(.991)$ \\
\hline
\end{tabular}

Note-In parentheses are the estimates of $B$ and $R^{2}$ when $A$ is fixed at .038 .
The parameter estimates were also obtained using a value of $\sqrt{ } A$ fixed at .038 . Having thus the same Weber fraction for counting, $\beta_{2}$, for all conditions, led to very good predictions. The best-fitting values for $B$, as well as for the $R^{2}$, are those reported in Table 10 in parentheses. These values for $B$ are slightly larger for the filled intervals than for the empty intervals, and are very much larger for visual than for auditory intervals. Thus, the data reveal that there is a countermechanism, $\sqrt{ } A$ or $\beta_{2}$, common to the different marker-type conditions, and an error in variance, as denoted by $B$, that is large for the visual mode but not for the auditory mode.

In brief, Experiment 5 yields some interesting results. First, the present findings are of theoretical importance because they support the conclusion, as in Fetterman and Killeen (1992) or as in Getty (1975), that a generalized form of Weber's law is relevant for time (for review, see Allan, 1979; or Killeen \& Weiss, 1987). In the present case, the demonstration is especially interesting for involving different marker-type conditions. Secondly, for the 250 - and 500 -msec intervals, the performance for both modalities is generally superior with empty intervals. Finally, there is no increment of the Weber fraction for $T$ values longer than $2 \mathrm{sec}$ as sometimes reported elsewhere for duration (Allan, 1979; Fraisse, 1978).

\section{GENERAL DISCUSSION}

The results observed in these five experiments show that the differences between performance with filled and empty intervals depend on the type of markers, on the range of duration investigated, and, to some extent, on the type of method employed for discriminating those intervals. To these sources of dependence influencing the levels of performance we could add also the large individual differences. Nonetheless, it is possible to draw some definite conclusions from the results.

In Experiments 1 and 2, that is, with the 250- and 50msec intervals, respectively, as in Experiment 4, the results for the visual modality clearly show better performance with the empty intervals than with the filled intervals. With the auditory modality, performance is not systematically better with the empty than with the filled intervals. At $50 \mathrm{msec}$, no filled/empty difference is ob- 


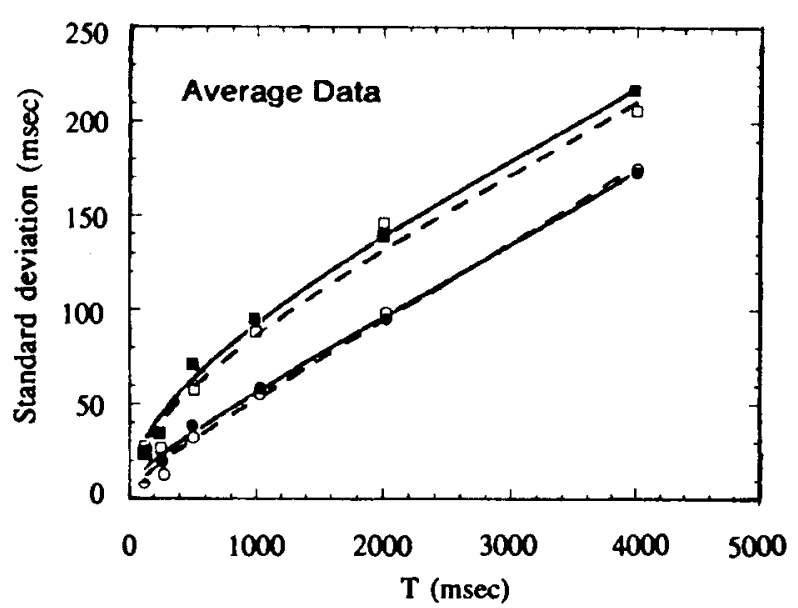

Figure 2. Standard deviation as a function of duration, $T$, for the average data of the 4 subjects of Experiment 5. Open circles = empty auditory intervals, filled circles $=$ filled auditory intervals, open squares = empty visual intervals, filled squares = filled visual intervals.

served (Experiments 2 and 4). At $250 \mathrm{msec}$, discrimination is better with empty intervals (Experiments 1 and 3), except with an adaptive procedure (Experiment 4). Also, as shown in Experiment 3, discrimination of duration in the auditory modality is easier with the FC method than with the SS method. In Experiment 5, the comparison of filled and empty intervals was extended to a wider range of durations: for short intervals, in the range of .25 to $.50 \mathrm{sec}$, the discrimination for empty intervals is generally easier than the discrimination for filled intervals. This difference fades for longer intervals. Although these results do not permit us to demonstrate the functioning of a timing process in duration discrimination, they help to question the relevance of some hypotheses about durationdiscrimination mechanisms.

Rammsayer and Lima (1991) observed that the discrimination of 50-msec intervals in the auditory mode was better with filled intervals than with empty intervals. This observation led them to propose different hypotheses about the mechanism of timing. One hypothesis suggests that the presence of more perceivable physical stimuli with filled intervals than with empty intervals results in an increased rate of neural firing of pulses on which the subjective representation of duration is based. The idea, that subjective duration is a product of an accumulation of internal pulses, is central to the classical psychophysical models of time (Creelman, 1962; Treisman, 1963). A higher rate of neural firing would result in a finer temporal resolution. Another hypothesis reported by Rammsayer and Lima stipulates that the superior discrimination observed with filled intervals might depend on the quality of the switch mode in a "pacemaker-switch-accumulator" process model of timing (Church, 1984; Gibbon \& Church, 1984; Meck, 1984). The filled intervals would benefit from a simpler, and consequently more efficient, switch mode than the empty intervals.
For both hypotheses-increased rate of neural firing and the switch mode-it could be predicted that whatever the sensory modality for providing the signals, the use of filled intervals should lead to better performance than the use of empty intervals. That prediction was shown to be wrong in the visual modality in both Experiment 1 and Experiment 2, that is, at 250 and $50 \mathrm{msec}$, and in Experiment 4 with the adaptive procedure. Thus, it is certainly not possible to generalize the findings of Rammsayer and Lima (1991) regarding the difference between filled and empty intervals. Our results reveal that there are several factors that may affect duration discrimination, in some cases leading to better discrimination with empty intervals than with filled intervals. What are some of those potential factors?

To begin with, it cannot be excluded that duration discrimination might be based on different dimensions according to the range of durations investigated and the experimental procedure employed. The upward slopes reported in Figure 1 at various points could be an indication that, at least for short durations, the time judgments, instead of being based on some temporal information, actually rely on a surrogate mechanism such as sensory integration. For very short intervals, it is already known that the physical characteristics of the markers influence duration discrimination (Allan, 1979). Moreover, in the case of empty intervals, the possibility that performance relies on some grouping principle, or perceptual organization, cannot be discarded. The possibility that there are different mechanisms for estimating time is also suggested by Rammsayer and Lima (1991), who have reported that discrimination of $1-\mathrm{sec}$ intervals is cognitively influenced whereas discrimination of 50 -msec intervals is perceptual in nature. Even within the range of very short intervals, there might be more than one mechanism for processing temporal information (Rammsayer, 1992a).

In any attempt to theorize about timing processes operating with different marker-type conditions, one has to deal with the superiority, with short durations, of the empty intervals over the filled intervals observed with the visual mode and in some conditions with the auditory mode. In explaining why, for a given range of durations, discrimination of empty intervals can be superior to the discrimination of filled intervals, the following tentative and simple explanation can be advanced. Let us call it the internal-marker hypothesis, which is in line with the onset-offset model of Allan, Kristofferson, and Wiens (1971). This hypothesis assumes that the time needed to eliminate the internal trace of a physical signal is longer than the time needed to generate an internal onset from a physical signal. This seems reasonable considering, for example, the different estimates of visual persistence (Nisly \& Wasserman, 1989). Thus, the hypothesis supposes that the internal onset and offset signals differ considerably with empty and filled conditions. With the filled intervals, the timing would start as soon as the physical signal was converted into an internal onset signal, and the internal offset would occur when the trace of the physi- 
cal signal disappeared. With the empty intervals, the internal onset might well occur after the disappearance of the trace of the first marker, and the internal offset would occur as soon as the second physical signal was detected. Consequently, the duration between the internal onset and offset of a filled interval would be longer than the duration between the internal onset and offset of an empty interval. This is consistent with Craig's (1973) findings, which suggest that a constant value must be added for an empty interval to be judged to be as long as a filled interval. In other words, we would have, between the short and the long intervals, the same $\Delta t$ difference, but the internal length of these intervals would be smaller in the empty conditions. The discrimination should then be easier with empty intervals, since, following Weber's law, the $\Delta t$ must increase with the increase of duration for reaching comparable levels of discrimination. This relative effect of the markers would eventually disappear as the duration became very long. For very short durations, this effect would be reflected in performance as soon as a more efficient process, such as sensory integration (Rammsayer \& Lima, 1991), was no longer available.

In brief, the internal-marker hypothesis is quite attractive for its simplicity, for its power to account for the difference between performance with filled and empty intervals observed in the different experiments of this study, and even for its capacity to account for the filled-duration illusion (Goldfarb \& Goldstone, 1963; Goldstone \& Goldfarb, 1963). Moreover, assuming that sensory persistence is longer for vision than for hearing, the hypothesis predicts that the difference between empty and filled intervals should prevail more with the visual mode than with the auditory mode, which is also consistent with the data obtained in this study.

In conclusion, the various findings of this study do not allow for the establishment of a general model for time perception, although they do provide many indices for any eventual theorizing effort. Experiment 5 (see in particular Figures $1 \mathrm{~b}$ and $1 \mathrm{c}$ ) shows that the generalized Weber's law holds for different marker-type conditions. This opens the door for a general theory of timing based on a single-timer assumption. The internal-marker hypothesis described above is an attempt to explain some data within this single-timer perspective. On the other hand, for very short intervals, there seems to be some process, such as sensory integration or perceptual organization, that provides a base for time judgments and that is very sensitive to the variations of the physical characteristics of the stimuli marking the intervals to be discriminated. More studies are certainly needed to determine the physical characteristics under which the different timing processes are most efficient, and to determine why this efficiency changes with psychophysical method. Moreover, certain upward shifts in the functions reported in Experiment 5 (see in particular Figures 1a and 1d) also invite us to do more specific work on identifying the most efficient strategy, such as explicit counting, that an ob- server may adopt for any given range of durations in a timing task.

\section{REFERENCES}

ABEL, S. M. (1972a). Discrimination of temporal gaps. Journal of the Acoustical Society of America, 52, 519-524.

ABEL, S. M. (1972b). Duration discrimination of noise and tone bursts. Journal of the Acoustical Society of America, 51, 1219-1223.

Allan, L. G. (1979). The perception of time. Perception \& Psychophysics, 26, 340-354.

Allan, L. G., Kristofferson, A. B. (1974). Psychophysical theories of duration discrimination. Perception \& Psychophysics, 16, 26-34.

Allan, L. G., Kristofferson, A. B., \& Rice, M. E. (1974). Some aspects of perceptual coding of duration in visual duration discrimination. Perception \& Psychophysics, 15, 83-88.

Allan, L. G., Kristofferson, A. B., Wiens, E. W. (1971). Duration discrimination of brief light flashes. Perception \& Psychophysics, 9, 327-334.

CARBOTte, R. M. (1972). Discrimination of brief empty time intervals. Unpublished doctoral dissertation, McMaster University.

CarbotTe, R. M. (1973). Retention of time information in forced-choice duration discrimination. Perception \& Psychophysics, 14, 440-444.

Сhurch, R. M. (1984). Properties of the internal clock. In J. Gibbon \& L. G. Allan (Eds.), Annals of the New York Academy of Science: Vol. 423, Timing and time perception (pp. 566-582). New York: New York Academy of Sciences.

Craig, J. C. (1973). A constant error in the perception of brief temporal intervals. Perception \& Psychophysics, 13, 99-104.

Creelman, C. D. (1962). Human discrimination of auditory duration. Journal of the Acoustical Society of America, 34, 582-593.

Creelman, C. D., Macmillan, N. A. (1979). Auditory phase and frequency discrimination: A comparison of nine procedures. Joumal of Experimental Psychology: Human Perception \& Performance, 5 , 146-156.

Divenyi, P. L., \& DANner, W. F. (1977). Discrimination of time intervals marked by brief acoustic pulses of various intensities and spectra. Perception \& Psychophysics, 21, 125-142.

DiVENYI, P. L., \&ACHS, R. M. (1978). Discrimination of time intervals bounded by tone bursts. Perception \& Psychophysics, 24, 429-436.

Fetterman, J. G., \& Killeen, P. R. (1990). A componential analysis of pacemaker-counter timing systems. Journal of Experimental Psychology: Human Perception \& Performance, 16, 766-780.

Fetterman, J. G., \& Killeen, P. R. (1992). Time discrimination in Columba livia and Homo sapiens. Joumal of Experimental Psychology: Animal Behavior Processes, 18, 80-94.

Fraisse, P. (1952). La perception de la durée comme organisation du successif. Année Psychologique, 52, 39-46.

Fraisse, P. (1978). Time and rhythm perception. In E. Carterette \& M. Friedman (Eds.), Handbook of perception VIII (pp. 203-254). New York: Academic Press.

GeTTY, D. J. (1975). Discrimination of short temporal intervals: A comparison of two models. Perception \& Psychophysics, 18, 1-8.

Gibbon, J., \& ChURCh, R. M. (1984). Sources of variance in an information processing theory of timing. In $\mathrm{H}$. L . Roitblat, $\mathrm{T}$. C. Bever, \& H. S. Terrace (Eds.), Animal cognition (pp. 465-488). Hillsdale, NJ: Erlbaum.

GoldFarb, J. L., \& Goldstone, S. (1963). Time judgment: A comparison of filled and unfilled durations. Perceptual \& Motor Skills, 16, 376 .

Goldstone, S., \&oldFARB, J. L. (1963). Judgment of filled and unfilled durations: Intersensory factors. Perceptual \& Motor Skills, 17, 763-774.

GoODFELlow, L. D. (1934). An empirical comparison of audition, vision, and touch in the discrimination of short intervals of time. American Journal of Psychology, 46, 243-258.

GreEN, D. M., \& SWETS, J. A. (1966). Signal detection theory and psychophysics. New York: Wiley. 
Grondin, S., \& Rousseau, R. (1991). Judging the relative duration of multimodal short empty time intervals. Perception \& Psychophysics, 49, 245-256.

HENRY, F. M. (1948). Discrimination of the duration of a sound. Journal of Experimental Psychology, 38, 734-743.

KAERNBACH, C. (1991). Simple adaptive testing with the weighted updown method. Perception \& Psychophysics, 49, 227-229.

KILleEN, P. R. (1992). Counting the minutes. In F. Macar, V. Pouthas, \& W. Friedman (Eds.), Time, action and cognition: Towards bridging the gap (pp. 203-214). Dordrecht: Klüwer.

KILlEen, P. R., \& Weiss, N. A. (1987). Optimal timing and the Weber function. Psychological Review, 94, 455-468.

KIRK, R. E. (1982). Experimental design: Procedures for the behavioral sciences. Belmont, CA: Brooks/Cole.

KLUMPP, R. G., \& EADY, H. R. (1956). Some measurements of interaural time difference thresholds. Journal of the Acoustical Society of America, 28, 859-860.

KrUEgER, L. E. (1989). Reconciling Fechner and Stevens: Toward a unified psychophysical law. Behavioral \& Brain Sciences, 12, 251-320.

MEck, W. H. (1984). Attentional bias between modalities: Effect on the internal clock, memory, and decision stages used in animal time discrimination. In J. Gibbon \& L. G. Allan (Eds.), Annals of the New York Academy of Sciences: Vol. 423. Timing and time perception (pp. 528-541). New York: New York Academy of Sciences.

Nisly, S. J., \& Wasserman, G. S. (1989). Intensity dependence of perceived duration: Data, theories, and neural integration. Psychological Bulletin, 106, 483-496.

RAMMSAYER, T. H. (1992a). Effects of marker duration on temporal information processing of empty auditory intervals. In G. Borg \& G. Neely (Eds.). Fechner Day 92: Proceedings of the Eighth Annual Meeting of the International Society for Psychophysics, Stockholm.
RAMmSAyer, T. H. (1992b). An experimental comparison of the weighted up-down method and the transformed up-down method. Bulletin of the Psychonomic Society, 30, 425-427.

Rammsayer, T. H., \& Lima, S. D. (1991). Duration discrimination of filled and empty auditory intervals: Cognitive and perceptual factors. Perception \& Psychophysics, 50, 565-574.

Rousseau, R., Poirier, J., \& Lemyre, L. (1983). Duration discrimination of empty time intervals marked by intermodal pulses. Perception \& Psychophysics, 34, 541-548.

Small, A. M., \& CAMPbell, R. A. (1962). Temporal differential sensitivity for auditory stimuli. American Journal of Psychology, 75, $401-410$.

Treisman, M. (1963). Temporal discrimination and the indifference interval: Implications for a model of the "internal clock." Psychological Monographs, 77 (Whole No. 576).

\section{NOTE}

1. In order not to overestimate the differential threshold in Experiment 5 , which would result in an underestimation of sensitivity, the following procedure was applied. If the subject had only 1 or 0 error for 1 point of the psychometric function, the next point that would logically be expected to be easier was not included in the analysis. For the cases in which there were 0 or 60 long responses out of 60 , the $Z$ values included in the function were, respectively, $-2.38(1 \%)$ and $2.38(99 \%)$. Excluded cases were more frequent with longer durations. There were at least 4 points remaining for each psychometric function.

(Manuscript received July 24, 1992; revision accepted for publication February 15, 1993.) 